\title{
Start-to-end simulations for a seeded harmonic generation free electron laser
}

\author{
S. Thorin, * M. Brandin, and S. Werin \\ MAX-lab, Lunds Universitet, P.O. Box 118, SE-221 00 Lund, Sweden \\ K. Goldammer ${ }^{\dagger}$ and J. Bahrdt \\ BESSY GmbH, Albert-Einstein-Straße 15, 12489 Berlin, Germany
}

(Received 19 June 2007; published 13 November 2007)

\begin{abstract}
This paper shows how the MAX linac injector and transport system can be efficiently retuned to suit free electron laser (FEL) performance. In a collaboration between MAX-lab and BESSY, a seeded harmonic generation free electron laser is being constructed at MAX-lab. The setup uses the existing MAX-lab facility upgraded with a new low emittance photocathode gun, a Ti:Sa $266 \mathrm{~nm}$ laser system used for both the gun and seeding and an FEL undulator system. To produce the high quality electron beam needed, it is shown how the magnet optics in an achromatic dogleg can be tuned to create an optimum bunch compression and how a good quality beam can be maintained through the beam transport and delivered to the FEL undulators. In extensive start-to-end simulations from the cathode of the gun to the generation of photons in the undulators, FEL performance and stability has been calculated using simulation tools like ASTRA, ELEGANT, and GENESIS. This has been done for both the third and fifth harmonic of the seed laser. The results from the calculation are $30 \mathrm{fs}$ light pulses with a power of $11 \mathrm{MW}$ at $88 \mathrm{~nm}$ and $1.4 \mathrm{MW}$ at $53 \mathrm{~nm}$.
\end{abstract}

DOI: 10.1103/PhysRevSTAB.10.110701

PACS numbers: 41.60.Cr, 41.85.Ew, 41.85.Lc, 41.75.Ht

\section{INTRODUCTION}

The creation of short, intense, and coherent radiation pulses with the development of free electron lasers is an important step for future light sources. The free electron laser (FEL) interaction, though, requires a high quality electron beam with high peak current, low energy spread and low emittance, and therefore a high performance source.

In collaboration between the synchrotron radiation laboratory MAX-lab in Lund, Sweden and the Berliner Elektronenspeicherring Gesellschaft für Synchrotronstrahlung (BESSY) in Berlin, Germany, a test facility for a seeded harmonic generation (HG)-FEL [1] will be built over the coming year at the MAX-laboratory in Lund. The test facility uses the existing MAX injector and provides an opportunity for investigating the design and function of various aspects of the FEL and also for testing simulation codes and scripts. The project also shows how a synchrotron radiation facility can be upgraded and used for FEL research.

The MAX injector [2] with its capabilities of up to $500 \mathrm{MeV}$ electrons will be used with a new low emittance photocathode gun installed. The gun is currently under construction and applies state-of-the art photoinjector technology to provide for high bunch charges and low beam emittance [3]. Using an off crest rf phase in the linacs and the magnetic optics in a translating dogleg in the transport

\footnotetext{
*sara.thorin@maxlab.lu.se

†goldammer@bessy.de
}

line, longitudinal compression of the electron bunch can be achieved. Since the compression is done after final acceleration the electron bunches have a relatively high energy spread. The rf phase and compression therefore have to be optimized with final energy spread as a more important factor than high current in order to get maximum light output power. The FEL interaction is seeded with a $500 \mathrm{fs}$, $266 \mathrm{~nm}$ laser pulse and the undulators are tuned to give radiation at either the third or fifth harmonic which corresponds to 88 and $53 \mathrm{~nm}$.

Simulating the different parts of the machine using different codes lets us do the right approximations for each part and conversion of the output using MATLAB makes it possible to automatically run a simulation through several codes. By doing start-to-end calculations [4] from the gun through the recirculator and transport to the end of the optical klystron, we will get an idea of the performance of the FEL and can optimize the whole setup on the final result, the radiation. It will also give us a chance to compare the simulation results with experiments.

\section{Layout of the project}

The FEL test facility collaboration is structured such that MAX-lab provides the high quality electron beam together with a seed laser for the experiment and BESSY will construct and install the FEL undulators. Electron and photon beam diagnostics as well as commissioning and operation of the FEL will be managed in close collaboration.

Apart from the new gun, the injector consists of two $5.2 \mathrm{~m}$ long linac structures each providing for a beam 


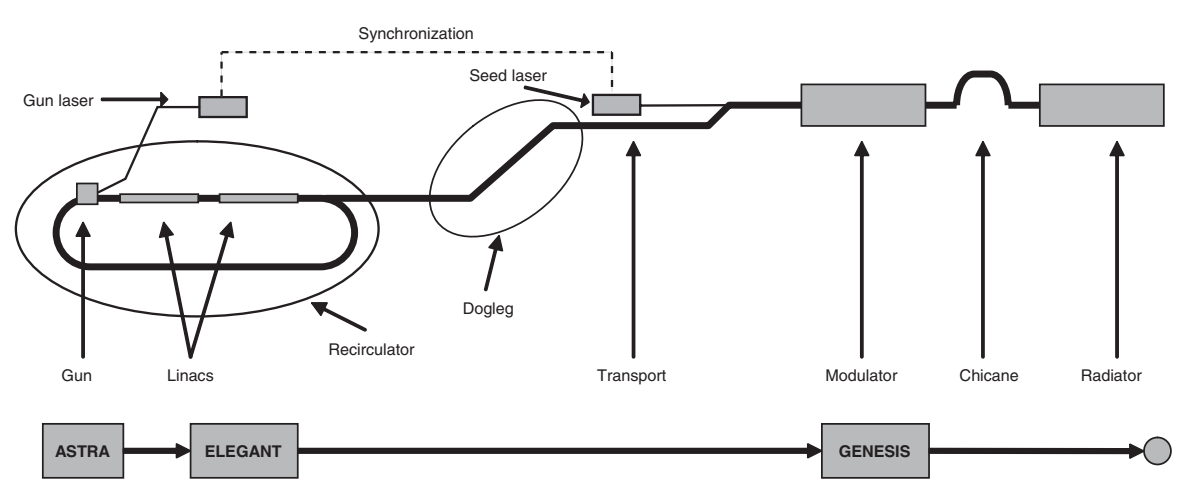

FIG. 1. Schematic view of the FEL setup and simulations.

energy of up to $125 \mathrm{MeV}$. When the electrons have passed both linacs they will be bent into a recirculator, turning them around 360 degrees and passing them through the linacs one more time. This gives a total beam energy of 400-500 MeV.

An achromatic dogleg is commonly used as a translating stage in beam transports and in this setup both compresses the electron bunches and elevates the beam from the cellar to ground level. The FEL undulators [5] are placed inside the MAX II ring, approximately $40 \mathrm{~m}$ from the injector. Just before the first undulator, the electrons will do a $20 \mathrm{~mm}$ translation so that a seed laser beam can be inserted.

The FEL section comprises one planar and one APPLE II type undulator and an intermediate magnetic chicane. This will make up a one stage HG-FEL, seeded with a Ti:sapphire laser at $266 \mathrm{~nm}$. A complete commercial laser system has been purchased from Thales SA and will be installed at MAX-lab. This laser will not only provide a $500 \mathrm{fs}, 100 \mu \mathrm{J}$ seeding pulse but will also give fully synchronized 10 ps pulses to be used for the photoinjector.

A schematic view of the FEL setup and the start-to-end simulations can be seen in Fig. 1.

\section{START-TO-END SIMULATIONS}

\section{A. The gun}

In order to produce a transversely and energetically collimated electron beam, the electrons are generated in a low emittance photocathode gun. The beam dynamics in the gun is simulated with the code ASTRA [6] which takes into account space charge effects. The gun is a $3 \mathrm{GHz}$ 1.6cell cavity, mounted slightly off-axis, with a copper cathode illuminated by a $10 \mathrm{ps}$ long Ti:Sa laser pulse. This produces bunches with a total charge of $0.5-1 \mathrm{nC}$. After the gun the electron beam is bent approximately $10 \mu \mathrm{rad}$ to go centered through the linac. This bend is simulated in ELEGANT [7] where a conversion of the output file between ASTRA and ELEGANT is done in MATLAB. The particle file is then converted back to ASTRA input and run through the first linac in the MAX-injector. The beam parameters at the exit from the gun can be seen in Table I and Fig. 2.

\section{B. The recirculator}

As mentioned earlier a recirculating system is used at MAX-lab to accelerate the electrons to their full energy (see Fig. 1). To make the two $180^{\circ}$ bending blocks, dipoles, quadrupoles, and sextupole magnets are used. The sextupoles are used to correct slightly for second order effects that appear from the sinusoidal shape of the accelerating field. These sextupoles are however already integrated into some of the quadrupoles and cannot be tuned separately and completely compensate for the second order effects.

The optics in the recirculator is such that the energy of the beam on the second turn through the linac has to be twice that of the first turn for the electrons to enter the transport line properly. The recirculator is simulated in ELEGANT.

TABLE I. Beam parameters after the gun.

\begin{tabular}{lc}
\hline \hline \multicolumn{1}{c}{ Energy } & $4 \mathrm{MeV}$ \\
\hline Energy spread & $3 \%$ \\
Normalized emittance & $3 \mathrm{~mm} \mathrm{mrad}$ \\
Pulse length (fwhm) & $11 \mathrm{ps}$ \\
Peak current & $45 \mathrm{~A}$ \\
Charge & $0.5 \mathrm{nC}$ \\
\hline \hline
\end{tabular}
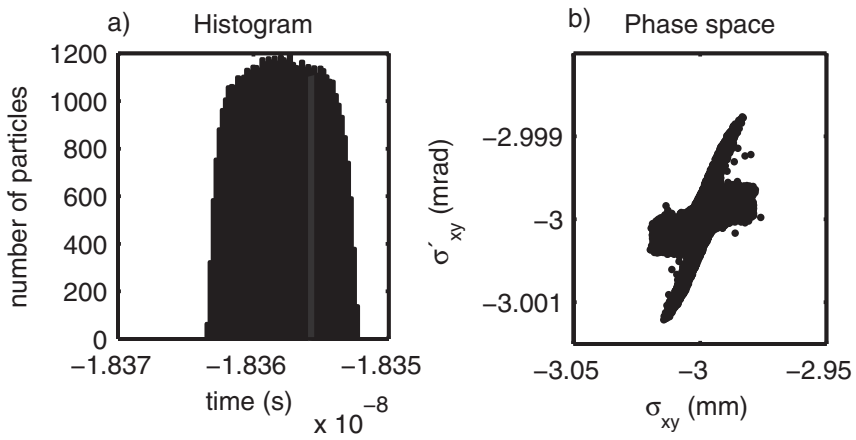

FIG. 2. Time structure (a) and transversal phase space (b) of the electron beam after the gun. 


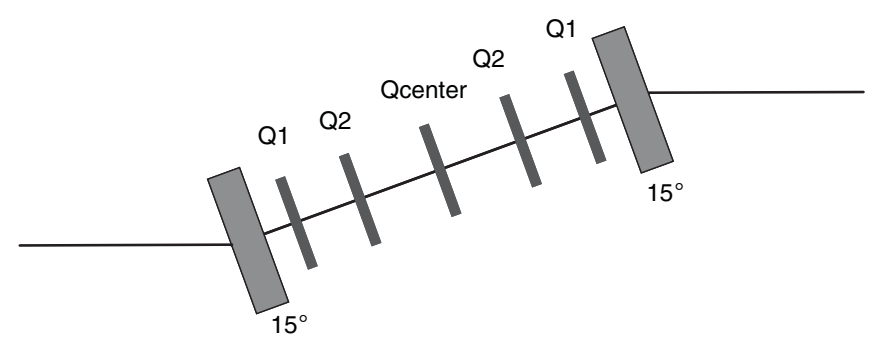

FIG. 3. Drawing of the translating dogleg where the bunch compression is done.

\section{Transport}

The transport from the injector to the undulator section is about $40 \mathrm{~m}$ and includes a vertical lift of the beam from the cellar to the ground floor, see Fig. 3. This lift is done with an achromatic dogleg consisting of two $15^{\circ}$ bends with 5 quadrupoles in between. The design of the dogleg is such that the middle quadrupole is used to control the beta function while the two outer ones are used to close dispersion after the second bend. The two quads on either side of the middle could be used to keep the beta function from getting a too small minimum in the center of the dogleg which potentially could introduce space charge effects. In this case ASTRA simulations show that space charge effects are negligible at this point and the quads are used to help create a beta focus at the right point in the modulator. The dogleg also introduces an $R_{56}$ suitable for compressing the electron bunch. The transport and compression is simulated in ELEGANT which takes coherent synchrotron radiation effects into account.

Near the end of the transport section there is a half chicane used to align the seed laser pulse with the electron beam. This bend is done upwards, which is in the bending plane of the electrons in the undulator, and gives a small dispersion to the beam in the $y$ direction. It is however small enough to not influence the beam size noticeably.

The optics in the transport is set to give a beam waist inside the first FEL undulator. The transversal rms size of the beam at the minimum is in the order of $250 \mu \mathrm{m}$, which corresponds to the size of the seed laser at this point.
The resultant beta and dispersion functions after optimization can be seen in Fig. 4 .

\section{Bunch compression}

Since a high peak current is important for FEL efficiency, the electron bunches have to be compressed after the gun. In this already existing machine there is no room to install a compressor chicane, but the dogleg in the transport can be used as a bunch compressor. The method is similar to the common bunch compression schemes in linear accelerators [8]: the electric field in the linac is given such a phase that an energy chirp is induced in the electron pulses. Through the quadrupoles and dipoles in the transport dogleg, this chirp can then be rotated timewise to result in a very short pulse. Optimizations, matching, and calculations of $R_{56}$, dispersion and Twiss parameters were made partially in DIMAD [9] and partially by hand through a graphical user interface written in MATLAB that lets you change the optics along the machine and check the corresponding Twiss parameters and $R_{56}$.

After optimizing the dogleg for both optics and compression, it gives an $R_{56}$ of $5.5 \mathrm{~cm}$ which means that an accelerating phase of $30^{\circ}$ would be ideal and give the best compression. However, in the recirculating system in the MAX-lab injector the electrons have to have twice the energy on the second pass though the linacs compared to the first pass in order to be extracted into the transport line. This gives the condition that the accelerating phase has to be the same in both passes though the linacs and taking dispersion, beam size, and energy spread into account a much lower accelerating phase had to be used. After investigating a series of cases a phase of 8 degrees was found to be optimal. This optimization was made to get the highest output laser power on the third harmonic. Approximately $20 \%$ of the bunch charge can be confined within a $300 \mathrm{fs}(90 \mu \mathrm{m})$ long part of the bunch with a mean current of $300 \mathrm{~A}$. The peak current goes up to $1.3 \mathrm{kA}$ in the main peak but in this region the energy spread is quite high $(\Delta \gamma / \gamma=0.25 \%)$. There is also a few peaks with lower current and lower energy spread of about $700 \mathrm{~A}$ and $\Delta \gamma / \gamma=0.09 \%$. The reason for the high energy spread is

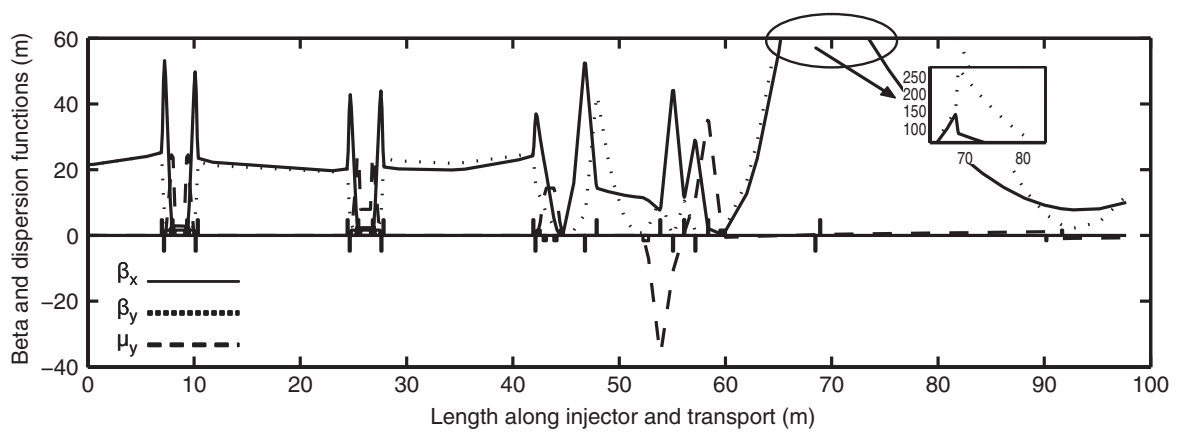

FIG. 4. Twiss parameters. The solid line shows the beta function in the nonbending plane. The dotted and dashed lines show the beta and dispersion functions in the bending plane. 

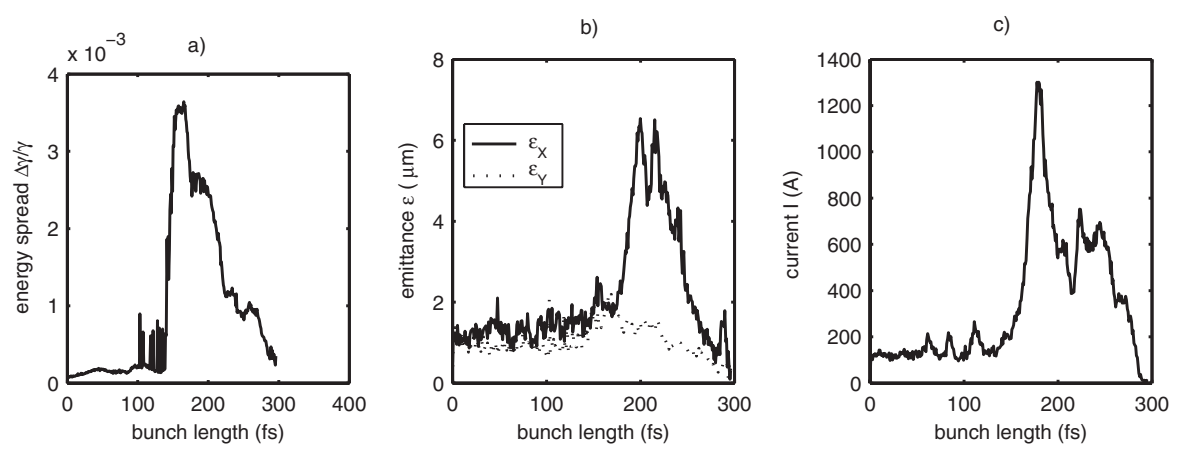

FIG. 5. Sliced energy spread (a), emittance (b), and current (c) just before the undulator section.

that all compression is done after acceleration, due to the layout of the injector facility, which means that the correlated energy spread (chirp) from the linac does not go through any subsequent acceleration and will thus not be lowered relative to the total energy. Table II gives the beam parameters and Fig. 5 show a slice analysis of the beam at the exit of the beam transport.

The demands on the electron beam before entering the undulators are low sliced emittance, low sliced energy spread, high peak current, and matched transverse size. The output from ELEGANT is converted using MATLAB into a format compatible with GENESIS 1.3 where the FEL simulations $[10,11]$ are done.

\section{FEL undulator section}

The FEL section comprises two undulators and an intermediate magnetic chicane. In the first undulator, called modulator, the electron beam copropagates with a strong seed laser of $266 \mathrm{~nm}$ wavelength and is modulated in energy. The particles then pass through the magnetic chicane which serves as a dispersive element. It consists of four dipole magnets and introduces an energy-dependent longitudinal delay of the electrons: the unmodulated particles are bent into a longer trajectory than the higher energy particles so that the beam is redistributed longitudinally. The process is referred to as "(micro)bunching." The chicane can be tuned to give different bunching at the entrance to the radiator which is needed to give maximum light output for either the resonant frequency of the modulator or a higher harmonic (harmonic generation). In the

TABLE II. Beam parameters of the compressed electron bunch just before entering the FEL undulators.

\begin{tabular}{lc}
\hline \hline & First peak (second peak) \\
\hline Energy & $400 \mathrm{MeV}$ \\
Energy spread & $0.25 \%(0.09 \%)$ \\
Normalized emittance & $4 \mathrm{~mm} \mathrm{mrad}(4 \mathrm{~mm} \mathrm{mrad})$ \\
Pulse length (fwhm) & $30 \mathrm{fs}(30 \mathrm{fs})$ \\
Peak current & $1.3 \mathrm{kA}(700 \mathrm{~A})$ \\
Charge & $0.04 \mathrm{nC}(0.02 \mathrm{nC})$ \\
\hline \hline
\end{tabular}

MAX-lab FEL project, the third harmonic will be used, thus efficiently shortening the output wavelength to $88 \mathrm{~nm}$. In the second undulator, called radiator, the bunched beam will then emit intense, coherent radiation at the shorter wavelength with an output power in the megawatt range. The 6-dimensional phase space file from ELEGANT is converted into a GENESIS input file by cutting out the seeded part of the beam and splitting it up into a collection of temporal slices. For each slice, the relevant beam parameters such as the average current, emittance, centroid, and beam size are calculated externally and delivered to a GENESIS compatible input file.

\section{E. FEL simulation}

The FEL consists of two different undulators as detailed in the previous section. Table III lists the properties of both undulators and the intermediate magnetic chicane.

The undulator parameter $\mathrm{K}$ was adjusted to yield optimal energy modulation in the modulator and a high output power in the radiator.

Time-dependent GENESIS simulations were performed to estimate the FEL output properties. At $88 \mathrm{~nm}$, the temporal

TABLE III. Parameters of the undulator section of the MAXlab-BESSY FEL when tuning the radiator to the 3rd harmonic $(88 \mathrm{~nm})$ of the seed laser. In parentheses, the properties for the 5 th harmonic $(53 \mathrm{~nm})$ are also given.

88 nm (53)

\section{Modulator}

Period length

Number of periods

K (rms)

Chicane (4 magnets)

Length of magnets

$12 \mathrm{~cm}$

Length of drifts

$40 \mathrm{~cm}$

Magnetic flux density

$12 \mathrm{mT}(8)$

Radiator

Period length

Number of periods

$56 \mathrm{~mm}$

30

$\mathrm{K}$ (rms)

$1.05(0.49)$

Peak output power 

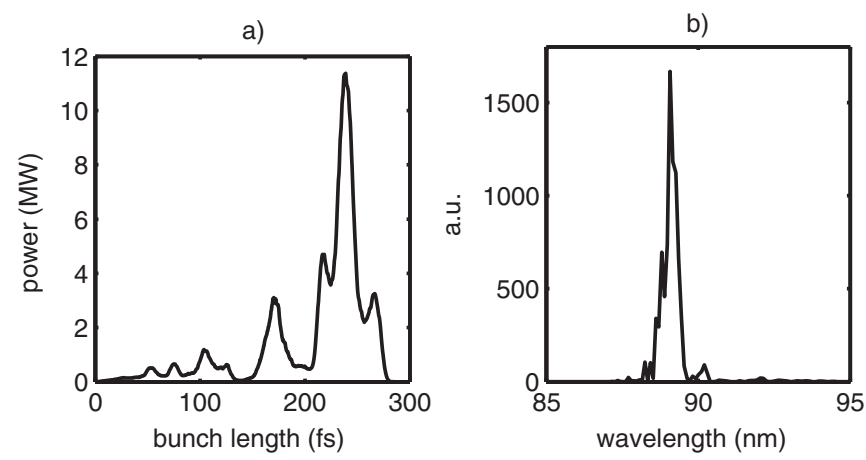

FIG. 6. Distribution of power along the bunch (a), and spectral power distribution (b), at the end of the radiator tuned to the $3 \mathrm{rd}$ harmonic.
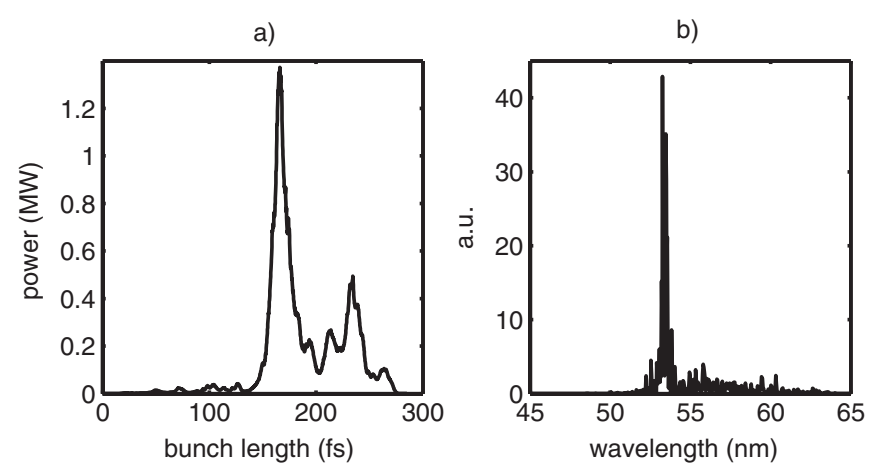

FIG. 7. Distribution of power along the bunch (a), and spectral power distribution (b), at the end of the radiator tuned to the 5th harmonic.

and spectral distribution of radiation power at the end of the radiator evolves as shown in Fig. 6. The corresponding output for the fifth harmonic, $54 \mathrm{~nm}$, can be seen in Fig. 7.

In fact, it becomes clear that at $88 \mathrm{~nm}$ the high current part of the beam lases with less intensity than the part with a low energy spread. This shows the relevance of the beam energy spread, as it counteracts microbunching in the magnetic chicane. At $55 \mathrm{~nm}$ however, all beam slices appear to lase equally well independent of their energy distribution, such that the temporal power profile closely resembles the current profile.

\section{CONCLUSION}

The FEL test facility at MAX-lab aims at reaching an output wavelength of $88 \mathrm{~nm}$ with a seeded HG-FEL. Startto-end tracking simulations show that the generation, compression, and transport of the beam from the existing MAX-lab injector to the FEL undulators can be done with a good electron beam quality maintained. In fully time-dependent FEL simulations, stable radiation at $88 \mathrm{~nm}$ could be shown. Even harmonic generation at the fifth harmonic at $54 \mathrm{~nm}$ could be seen. The results from the beam dynamic calculations give a short spike of electrons with high current which lead to a laser pulse of $30 \mathrm{fs}$ duration and output power of $11 \mathrm{MW}$ for $88 \mathrm{~nm}$ and 1.4 MW for $54 \mathrm{~nm}$. Even higher laser power could have been achieved with a lower energy spread in the electron bunch after compression. To accomplish this, the existing facility at MAX-lab would have to be rebuilt so that the bunches are compressed before the final acceleration.

Wakefields were taken into account inside the accelerating structure, but they were not included through the electron transport where the effect is believed to be much smaller [12].

The overall results from the start-to-end simulation remain to be verified in experiments.

\section{ACKNOWLEDGMENTS}

This work has been partially supported by the EU Commission in the Sixth Framework Program, Contract No. 011935-EUROFEL, and the Swedish Research Council.

[1] L. H. Yu, Phys. Rev. A 44, 5178 (1991).

[2] S. Werin et al., Commissioning of the $500 \mathrm{MeV}$ Injector for MAX-lab, EPAC 2004.

[3] S. Werin et al., Design of a New Preinjector for the MAX Recirculator to be used in EUROFEL, EPAC 2006.

[4] M. Abo-Bakr et al., Nucl. Instrum. Methods Phys. Res., Sect. A 528, 476 (2004).

[5] J. Bahrdt et al., Undulators for a Seeded HGHG-FEL at MAX-lab, EPAC 2006.

[6] K. Flöttman, ASTRA User Manual, 2000, www.desy.de/ $\sim$ mpyflo.

[7] M. Borland, APS Report No. LS-287, 2000.

[8] V. K. Bharadwaj et al., Linac Design for the LCLS Project at SLAC, PAC97.

[9] R. V. Servranckx, TRIUMF Design Note No. TRI-DN-93K233, 1993.

[10] S. Reiche, Nucl. Instrum. Methods Phys. Res., Sect. A 429, 243 (1999).

[11] C. Sung et al., Phys. Rev. ST Accel. Beams 9, 120703 (2006).

[12] S. D. M. Di Mitri et al., Electron Beam Simulations for the FERMI Project at ELETTRA, FEL Conference 2004, pp. 159-162. 